\title{
Cereal mycorrhiza: an ancient symbiosis in modern agriculture
}

\author{
Ruairidh J.H. Sawers, Caroline Gutjahr and Uta Paszkowski
}

Department of Plant Molecular Biology, University of Lausanne, Biophore Building, CH-1015 Lausanne, Switzerland

\begin{abstract}
The majority of terrestrial plants live in association with symbiotic fungi that facilitate mineral nutrient uptake. The oldest and most prevalent of these associations are the arbuscular mycorrhizal (AM) symbioses that first evolved $\sim 400$ million years ago, coinciding with the appearance of the first land plants. Crop domestication, in comparison, is a relatively recent event, beginning $\sim 10000$ years ago. How has the dramatic change from wild to cultivated ecosystems impacted $A M$ associations, and do these ancient symbioses potentially have a role in modern agriculture? Here, we review recent advances in $A M$ research and the use of breeding approaches to generate new crop varieties that enhance the agronomic potential of AM associations.
\end{abstract}

\section{Cereal crops retain an ancient ability to interact with AM fungi}

Arbuscular mycorrhizal (AM) symbioses are formed between plants and members of the Glomeromycota [1]. The fungus grows within the host roots, intermittently forming branched, tree-like structures, known as arbuscules, which are believed to be the main site of nutrient exchange [2]. In addition, the fungus develops an extensive network of extraradical hyphae that extends beyond the plant root system. Glomeromycotan fungi are obligate symbionts and rely on carbon provided by their plant hosts to complete their life cycle. In return, the fungus provides several benefits to the host plant, including improved mineral nutrition, enhanced drought tolerance and increased protection against pathogens [2]. The possible role of AM symbiosis in phosphate nutrition has received the most attention with regard to agricultural applications [3]. The recent discovery that nitrogen is also transferred by AM fungi has prompted further agronomic interest $[4,5]$.

AM symbioses first evolved $\sim 400$ million years ago [6,7], possibly helping the earliest terrestrial plants to take up nutrients in the absence of complex vascular root systems [8]. By contrast, the domestication of crop plants began relatively recently, $\sim 10000$ years ago [9]. At this point, a small segment of the available plant diversity was removed from its millennia-old environment and subjected to an intense period of selection, culminating in today's intensive agricultural practices. The evolutionary history and taxonomic distribution of AM interactions suggest that the progenitors of our modern cereal crops inherited the capacity to interact with Glomeromycotan fungi. Where

Corresponding author: Sawers, R.J.H. (ruairidh.sawers@unil.ch). investigated, this ability has been retained in modern cereal cultivars [10-13].

\section{Offsetting the costs of AM association}

For the plant host the cost of AM colonization is the delivery of $4-20 \%$ of photosynthetically fixed carbon to its fungal partner $[14,15]$. Historically, these costs must have been offset by the benefits to the colonized plant. If AM colonization is to be beneficial in an agronomic setting, the carbon demands must be compensated for by savings in crop production costs, the most attractive scenario being a reduction in the use of costly soil additives. AM symbioses are complex biological interactions; their impact varies in different environmental conditions and depends on the specific combination of plant and fungus involved [1618]. Consequently, profitable use of AM symbioses in an agricultural context requires the selection of a suitable combination of plant host, fungal partner and agricultural practice to balance costs and benefits. Here, we focus on the plant component of this problem and consider the question of identifying, or generating, plant varieties with an enhanced capacity to benefit from AM associations.

To successfully tailor a complex biological interaction, such as an AM symbiosis, for agronomic application using targeted manipulation requires a level of understanding that exceeds what is currently available. By contrast, an appreciation and understanding of existing variation can allow desirable traits to be directly selected with far less prior knowledge. In the following sections we discuss the potential and problems relating to isolating useful variation by comparing AM colonization between plant varieties as well as the impact recent physiological and molecular findings have had on this approach.

\section{Measuring performance differences following AM colonization}

The impact of AM colonization on a particular variety can be estimated by the difference in performance (measured as, for example, stature, total dry weight, yield) between colonized and non-colonized plants - defined as 'responsiveness'. (In earlier reports, there have been various uses of the terms 'response' and 'responsiveness'. The definition given here follows that proposed by Janos [19].) The relative performance of colonized and non-colonized plants (i.e. responsiveness) will change depending on environmental conditions [19] (Figure 1a). Importantly for agricultural applications, responsiveness tends to diminish as nutrient availability becomes saturating and colonization can impose a net performance cost under high nutrient 


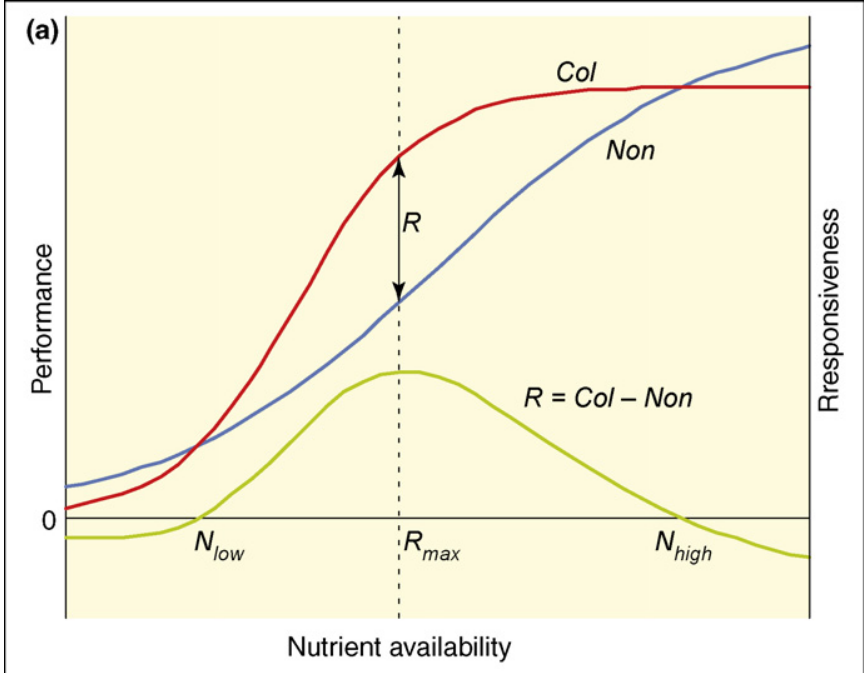

(b)

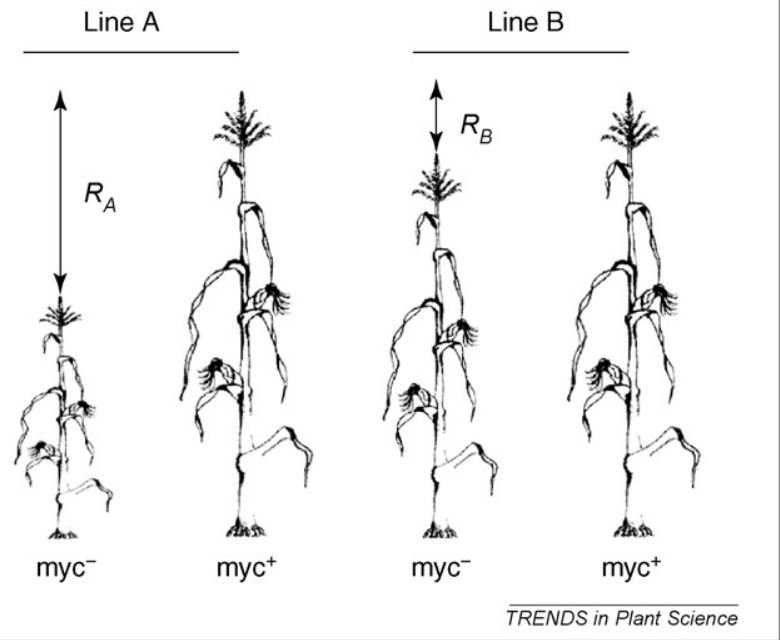

Figure 1. Calculating AM responsiveness. (a) When plant performance is measured with reference to a single changing nutrient, a sigmoidal relationship is observed [19]. Theoretical response curves for AM colonized ( $\mathrm{Col}$, red line) and non-colonized (Non, blue line) plants are shown. An arbitrary measure of plant performance is shown as a function of nutrient availability. Responsiveness $(R)$ is shown as the difference in performance between colonized and non-colonized plants (green line). Between a lower $\left(N_{\text {low }}\right)$ and higher level $\left(N_{\text {high }}\right)$ of nutrient, AM colonization is beneficial to plant performance (i.e. $R$ is positive). Maximum responsiveness is shown as $R_{\max }$ (b) Dependence effects can dominate responsiveness variation. Two varieties (Line $a$, Line b) that show growth differences in the absence of mycorrhiza $\left(\mathrm{myc}^{-}\right)$but perform equally well in the presence of mycorrhiza $\left(\mathrm{myc}^{+}\right)$will be considered to differ in mycorrhizal responsiveness $\left(R_{\mathrm{A}}, R_{\mathrm{B}}\right)$ although there is no difference with regards to the symbiosis itself. Such dependence effects can confound attempts to use responsiveness variation to isolate factors that impact the symbiosis directly.

conditions [12,19]. Any assessment of a given species or variety as 'responsive' or 'non-responsive' must always be qualified with reference to the growth conditions. This complication is magnified when a comparison is to be made between two or more varieties.

\section{Understanding diversity in AM responsiveness}

Although responsiveness is clearly defined for a single plant variety, comparing responsiveness between varieties becomes more problematic. First, to be meaningful, comparisons must be made under equivalent environmental conditions, taking into consideration that the relationship between absolute nutrient levels and relative performance might differ between varieties. In this respect, maximum responsiveness (the greatest difference between colonized and non-colonized plants when performance is measured across an environmental range) has been proposed as a suitable summary statistic [19] (Figure 1a). However, there are clear practical limitations in determining maximum responsiveness for a large collection of material or if multiple environmental variables are considered.

More fundamentally, responsiveness confounds several traits and it is misleading to consider high responsiveness alone as identifying the genetic basis of more profitable AM interactions. In certain varieties, high responsiveness is characterized as much by a good capacity to profit from AM colonization as by an inability to function in its absence. This point has been illustrated in the extreme case of the maize mutant lateral root1 (lrt1), a mutant deficient in lateral root growth. This mutant is highly intolerant of low phosphate conditions, but can be partially rescued by formation of an AM symbiosis [20]. Thus, it can exhibit higher responsiveness compared with wild-type siblings, although the greatest difference is in performance in the absence of AM colonization.

The inability of a plant to grow in the absence of colonization has been defined as 'dependence' and is calculated as the level of nutrient availability below which non-colonized plants either cease to grow or fall below a given performance threshold [19]. By extension, variation in responsiveness can be partitioned into dependence and non-dependence components. In a simple experimental system, dependence variation relates to plant performance under a given set of abiotic conditions. By contrast, nondependence variation describes differences in the interaction between plant and fungus. As such, non-dependence differences will include variation in the ability of lines to establish colonization, variation in the efficiency of nutrient uptake and variation in the regulation of nutrient exchange between fungus and host. These traits are of the greatest interest to the researcher aiming to enhance the profitability of cereal-AM interactions. If responsiveness is to be used to uncover useful variation in performance, dependence and non-dependence effects must be distinguished. A highly responsive line identified on the basis of exceptionally poor performance in the absence of AM colonization is unlikely to provide factors that maintain their positive effect following transfer into novel genetic backgrounds (Figure $1 \mathrm{~b}$ ).

\section{Existing variation in cereal responsiveness}

Several studies have investigated responsiveness variation present in existing crop diversity collections, usually with a focus on plant growth under limiting nutrient conditions. In a study comparing the performance of wheat varieties developed before and after 1900, varieties developed before 1900 were more responsive to AM colonization than those developed later [11] and it has been suggested that plant breeding has selected against AM association. However, in this study the observed differences in responsiveness were largely determined by an increased ability of modern lines to take up phosphate without AM fungi (i.e. a reduction in dependence, not a loss of compatibility with the fungus). Dependence is considered a greater target for 
natural selection than other components of responsiveness because it is based on plant factors alone and not on the interaction between plant and fungus [19]. The data presented in this study suggest that artificial selection has also acted strongly on dependence during the breeding of modern wheat varieties [11].

The confounding effect of dependence variation is also evident when assessing responsiveness differences among contemporary varieties. In a performance study of MidWestern US corn inbred lines, a range of responsiveness was observed [12]. However, when lines were selected for extremes of responsiveness and differences were investigated using quantitative trait locus (QTL) analysis, dependence differences were found to dominate [12]. From a breeding point of view, measuring responsiveness variation without consideration of dependence and non-dependence components will tend to uncover genetic components linked to the ability to grow in the absence of AM symbioses and not useful variation or factors that impact the symbiosis itself. However, advances in molecular and physiological understanding are helping to clarify the important distinction between dependence and non-dependence and are also extending the range of tools available for the identification of beneficial variation.

\section{The molecular basis of responsiveness variation}

Responsiveness, as a direct measure of AM contribution, considers the effect of AM colonization to be a simple addition to performance in the absence of colonization. This is most easily illustrated with regard to nutrient uptake; it is assumed that in the presence of the fungus the plant receives a nutrient supplement in addition to that which it would take up in the absence of colonization. Such an assumption is inherent in an attempt to identify variation that directly impacts AM symbioses on the basis of responsiveness alone. As described previously, dependence and non-dependence effects complicate the situation. Recent advances in molecular and physiological understanding further challenge the traditional interpretation, as can be exemplified by a brief discussion of plant phosphate uptake.

Plants use two distinct phosphate uptake pathways, one acquiring phosphate directly from the soil and one following delivery by the fungal partner (Box 1). Physiological studies have suggested that these two delivery modes might be considered functional alternatives, one of which will dominate depending on environmental conditions [18]. This distinction has been supported by expression analyses of the Phosphate transporter 1 (Pht1) family of phosphate transporters. Pht1 proteins are $\mathrm{H}_{2} \mathrm{PO}_{4}{ }^{-}-\mathrm{H}^{+}$symporters involved in the uptake of inorganic phosphate from the soil and the redistribution of phosphate within the plant [21]. Owing to the availability of a complete genome sequence, the Pht1 family of rice is the best characterized with respect to AM association [22]. The rice genome encodes a total of 13 Pht1 homologs (ORYsa:Pht1;1- ORYsa:Pht1;13), 10 of which have been shown to be expressed in roots [22]. ORYsa:Pht1;2 and ORYsa:Pht1;6 are highly expressed in the absence of AM association but are repressed following fungal colonization. Conversely, ORY$s a: P h t 1 ; 11$ is not expressed in the absence of the symbiosis

\section{Box 1. Phosphate uptake by AM plants}

In the majority of environments phosphorus is limiting for plant growth, owing, in part, to rapid immobilization of orthophosphate by free cations [42]. Consequently, large amounts of chemical phosphate fertilizers are added annually to cultivated soils [ $>4000000$ tons per year in the USA alone (United Nations Food and Agriculture organization; www.fao.org)], although the efficiency of uptake might be as low as $20 \%$ [43]. Phosphate is increasingly costly to extract and supplies are predicted to be exhausted before the end of this century. In addition, phosphate application presents severe environmental problems in the form of field run-off to water courses. The potential of AM symbioses to improve the way phosphate additions are used in agriculture is therefore of great interest.

Plants take up phosphate in two ways, either directly from the soil (the direct pathway, Figure la) or following delivery by a mycorrhizal fungal partner (the mycorrhizal pathway, Figure lb) $[3,18]$. Phosphate uptake via the direct pathway exceeds the rate of phosphate diffusion in the soil, generating a zone of phosphate depletion around the roots. The hyphal network of colonizing fungi reaches beyond this zone such that a greater soil area is available for uptake via the AM pathway. Phosphate is taken up by fungal extraradical hyphae [44-46] and translocated to arbuscules where it is released into the periarbuscular space and taken up via specific plant phosphate transporters (PTs) localized in the periarbuscular membrane $[31,47,48]$ (Figure I). The MEDtu:Pht1;4 protein of medic (Medicago truncatula) is the best-characterized AM symbiosisassociated plant PT and has been shown by genetic disruption to be involved in AM phosphate uptake $[31,47]$. Within the cereals, potential MEDtu:Pht1;4 orthologs have been identified from rice (ORYsa:Pht1;11) [22], maize (ZEAma:Pht1;6) [49], barley (HORvu:PHT1;8) [49] and wheat (TRlae:PHT1;myc) [50]. Although there is no functional evidence that these cereal PTs have a direct role in AM symbiosis, correlative expression data and sequence similarity to MEDtu:Pht1;4 suggest a common function.

(a)
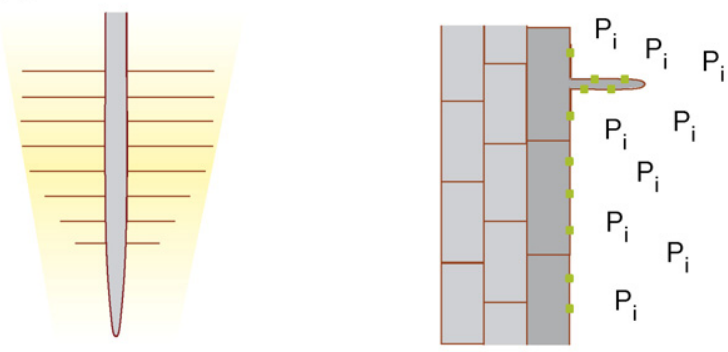

(b)
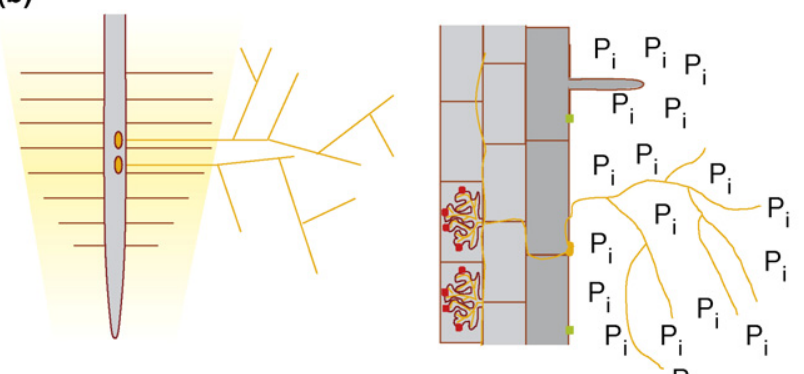

$\overline{T R E N D S}$ in Plant Science

Figure I. Phosphate uptake pathways. Phosphate $\left(\mathrm{P}_{\mathrm{i}}\right)$ uptake by (a) nonmycorrhizal and (b) mycorrhizal roots. In the absence of colonization (a), phosphate is taken up by plant phosphate transporters of the direct pathway (green) located in the outer membrane of the rhizodermis and root hairs. In mycorrhizal plants (b) a second pathway becomes active; phosphate is taken up by the extraradical hyphal network (orange) and delivered to arbuscles where it is absorbed by plant phosphate transporters (red) in the periarbuscular membrane. Following colonization, the accumulation of transcripts encoding the direct pathway transporters is reduced. 
but is strongly induced following AM colonization. These, and similar observations in other plant species, have revealed that distinct transporters are specific to the direct or the AM pathway (Box 1). It is evident that lines carrying alleles that contribute differently to the direct pathway might exhibit responsiveness differences independent of variation in components associated with the AM pathway.

When AM colonization is established there is not a simple superimposition of additional gene expression on the non-colonized state, but rather a switch from one pattern of gene expression to another - certain genes are induced and others repressed [22,23]. Thus, the genetic determinants of performance in AM-colonized and noncolonized plants can vary independently. It is tempting to speculate that the physiological and molecular distinction between the non-colonized and colonized state (for example, the direct and AM phosphate uptake pathways) underlies the distinction between dependence and non-dependence components of responsiveness. Importantly for studies of variation, colonized and non-colonized states are not simply additive and, consequently, non-colonized performance cannot provide a control or normalization-measure for crossvariety comparisons. One solution to this problem is refinement of the measure of AM impact that is used in diversity studies. A complementary approach is to use molecular information to directly investigate AM-associated variation.

\section{Molecular approaches for the identification of variation in AM symbioses}

Increased molecular characterization has opened up the possibility of applying molecular breeding techniques to the study of cereal-AM interactions. In addition to mycorrhizal PTs, several other cereal genes have now been implicated in the functioning of AM symbiosis, including potential cereal orthologs of the legume common symbiosis genes $[24,25]$ and candidates identified by expression profiling [23]. These genes present attractive targets for DNA sequencing approaches. Sequence information can be obtained from diverse varieties and directly examined for important amino acid changes on the basis of functional prediction or using the techniques of association analysis [26]. In addition, investigation of patterns of nucleotide change can reveal the strength of selective pressures acting on a given gene during domestication [27]. AM candidate genes also represent markers to be used for 'molecular phenotyping' in which gene expression is compared in different varieties to directly assay activity under certain conditions. Perhaps some of the most exciting possibilities involve a combination of molecular-genetic and diversity approaches. Reverse genetics techniques have the potential to identify loss-of-function alleles of AM candidate genes and provide material that could enable direct assessment of the importance of AM symbioses in agricultural systems. Characterization of such mutant alleles in highly responsive or non-responsive genetic backgrounds would then enable assessment of dependence and non-dependence variation.

\section{Moving towards an enhanced response to AM colonization?}

It is interesting to speculate as to what traits will be identified by selection for an enhanced AM response.
The profitable application of AM symbioses in cereal production does not demand large-scale qualitative genetic changes as would, for example, engineering of nodulation response or the introduction of $\mathrm{C}_{4}$ photosynthesis into $\mathrm{C}_{3}$ species. What is needed is a careful balancing of costs and benefits. Even if such a balance had been attained by the forces of evolution in a natural setting, it will have been greatly disrupted by the processes of domestication and contemporary agricultural practices. Moreover, even under natural conditions the exchange of nutrients need not be completely equitable and selective pressure can act on either partner to shift the balance to their advantage [28-30].

What is required for the cereal breeder is the ability to manipulate plant genetic factors that impact the costbenefit balance of an AM symbiosis, or more specifically the rate at which resources are diverted to the fungus in return for a given level of enhanced performance or reduction in production costs. There is increasing evidence that the plant host can regulate fungal colonization by modulating carbon delivery to the fungus [31,32] or by less direct signaling mechanisms [33,34], possibly involving phytohormones [35-38]. Selection for alterations in plant regulation of fungal growth is one way in which breeders might manipulate the cost-benefit balance of AM symbiosis. It has been shown that the degree of AM colonization of maize roots decreases as phosphate availability increases [39]. Similarly, barley has been shown to resist further AM colonization once a certain critical level of fungal growth is reached [40]. Selection of variation in such responses has the potential to improve the profitability of AM symbioses in a cultivated setting.

Crop diversity collections represent a valuable and powerful breeding resource. For example, modern cultivated maize lines retain up to $60 \%$ of the diversity present in ancestral teosinte (Zea mays spp. parviglumis) germplasm [41] and, at the nucleotide level, are more diverse than human, Drosophila or many wild-plant populations [26]. The use of such material requires clear understanding of the traits to be selected and benefits from the availability of molecular information. In this article, we have presented recent advances that are helping to fulfill these requirements for the study of AM symbioses and that should enable application of molecular breeding approaches to the optimization of AM symbioses for agricultural use. In achieving this, a 400 million-year-old interaction can be harnessed as a component of future agricultural systems.

\section{Acknowledgements}

We acknowledge the many workers we were unable to cite owing to space constraints, particularly those working outside the area of cereals who have made important contributions to the fundamental understanding of AM symbioses. We thank Ian Sanders (University of Lausanne) for discussion during the preparation of this article.

\section{References}

1 Schüssler, A. et al. (2001) A new fungal phylum, the Glomeromycota: phylogeny and evolution. Mycol. Res. 105, 1413-1421

2 Smith, S.E. and Read, D.J. (1997) Mycorrhizal Symbiosis, Academic Press

3 Karandashov, V. and Bucher, M. (2005) Symbiotic phosphate transport in arbuscular mycorrhizas. Trends Plant Sci. 10, 22-29 
4 Cruz, C. et al. (2007) Enzymatic evidence for the key role of arginine in nitrogen translocation by arbuscular mycorrhizal fungi. Plant Physiol. 144, 782-792

5 Govindarajulu, M. et al. (2005) Nitrogen transfer in the arbuscular mycorrhizal symbiosis. Nature 435, 819-823

6 Remy, W. et al. (1994) Four hundred-million-year-old vesicular arbuscular mycorrhizae. Proc. Natl. Acad. Sci. U. S. A. 91, 1184111843

7 Redecker, D. et al. (2000) Glomalean fungi from the Ordovician. Science 289, 1920-1921

8 Simon, L. et al. (1993) Origin and diversification of endomycorrhizal fungi and coincidence with vascular land plants. Nature 363, 67-69

9 Doebley, J.F. et al. (2006) The molecular genetics of crop domestication. Cell 127, 1309-1321

10 Koide, R. et al. (1988) Role of mycorrhizal infection in the growth and reproduction of wild vs. cultivated plants. I. Wild vs. cultivated oats. Oecologia 77, 537-543

11 Hetrick, B.A.D. et al. (1992) Mycorrhizal dependence of modern wheat variaties, landraces, and ancestors. Can. J. Bot. 70, 2032-2040

12 Kaeppler, S.M. et al. (2000) Variation among maize inbred lines and detection of quantitative trait loci for growth at low phosphorus and responsiveness to arbuscular mycorrhizal fungi. Crop Sci. 40, 358-364

$13 \mathrm{Zhu}$, Y-G. et al. (2001) Phosphorus (P) efficiencies and mycorrhizal responsiveness of old and modern wheat cultivars. Plant Soil 237, 249255

14 Wright, D.P. et al. (1998) Mycorrhizal sink strength influences whole plant carbon balance of Trifolium repens L. Plant Cell Environ. 21, 881-891

15 Bago, B. et al. (2000) Carbon metabolism and transport in arbuscular mycorrhizas. Plant Physiol. 124, 949-958

16 Johnson, N.C. et al. (1997) Functioning of mycorrhizal associations along the mutualism-parasitism continuum. New Phytol. 135, 575-586

17 Burleigh, S.H. et al. (2002) Functional diversity of arbuscular mycorrhizas extends to the expression of plant genes involved in $\mathrm{P}$ nutrition. J. Exp. Bot. 53, 1593-1601

18 Smith, S.E. et al. (2003) Mycorrhizal fungi can dominate phosphate supply to plants irrespective of growth responses. Plant Physiol. 133, $16-20$

19 Janos, D.P. (2007) Plant responsiveness to mycorrhizas differs from dependence upon mycorrhizas. Mycorrhiza 17, 75-91

20 Paszkowski, U. and Boller, T. (2002) The growth defect of $l r t 1$, a maize mutant lacking lateral roots, can be complemented by symbiotic fungi or high phosphate nutrition. Planta 214, 584-590

21 Smith, F. et al. (2003) Phosphate transport in plants. Plant Soil 248, 71-83

22 Paszkowski, U. et al. (2002) Rice phosphate transporters include an evolutionarily divergent gene specifically activated in arbuscular mycorrhizal symbiosis. Proc. Natl. Acad. Sci. U. S. A. 99, 13324-13329

23 Güimil, S. et al. (2005) Comparative transcriptomics of rice reveals an ancient pattern of response to microbial colonization. Proc. Natl. Acad. Sci. U. S. A. $102,8066-8070$

24 Paszkowski, U. et al. (2006) Maize mutants affected at distinct stages of the arbuscular mycorrhizal symbiosis. Plant J. 47, 165-173

25 Chen, C. et al. (2007) Fungal symbiosis in rice requires an ortholog of a legume common symbiosis gene encoding a $\mathrm{Ca}+$ /calmodulin-dependent protein kinase. Plant Physiol. 145, 1619-1628

26 Buckler, E.S. et al. (2006) Molecular and functional diversity of maize. Curr. Opin. Plant Biol. 9, 172-176

27 Tracy, W.F. et al. (2006) Recurrent mutation and genome evolution: Example of Sugary1 and the origin of sweet maize. Crop Sci. 46, S49-S54

28 Kiers, E.T. and van der Heijden, M.G. (2006) Mutualistic stability in the arbuscular mycorrhizal symbiosis: exploring hypotheses of evolutionary cooperation. Ecology 87, 1627-1636

29 Paszkowski, U. (2006) Mutualism and parasitism: the yin and yang of plant symbioses. Curr. Opin. Plant Biol. 9, 364-370
30 Sanders, I.R. (2003) Preference, specificity and cheating in the arbuscular mycorrhizal symbiosis. Trends Plant Sci. 8, 143-145

31 Javot, H. et al. (2007) A Medicago truncatula phosphate transporter indispensible for the arbuscular mycorrhizal symbiosis. Proc. Natl. Acad. Sci. U. S. A. 104, 1720-1725

32 Schaarschmidt, S. et al. (2007) Regulation of arbuscular mycorrhization by carbon. The symbiotic interaction cannot be improved by increased carbon availability accomplished by rootspecifically enhanced invertase activity. Plant Physiol. 143, 1827-1840

33 Staehelin, C. et al. (2001) Medicago truncatula plants overexpressing the early nodulin gene Enod40 exhibit accelerated mycorrhizal colonization and enhanced formation of arbuscules. Proc. Natl. Acad. Sci. U. S. A. 98, 15366-15371

34 Fitter, A.H. (2006) What is the link between carbon and phosphorus fluxes in arbuscular mycorrhizas? A null hypothesis for symbiotic function. New Phytol. 172, 3-6

35 Fitze, D. et al. (2005) Auxins in the development of an arbuscular mycorrhizal symbiosis in maize. J. Plant Physiol. 162, 1210-1219

36 Herrera-Medina, M.J. et al. (2007) Absicic acid determines arbuscule development and functionality in the tomato arbuscular mycorrhiza. New Phytol. 175, 554-564

37 Meixner, C. et al. (2005) Lack of mycorrhizal autoregulation and phytohormonal changes in the supernodulating soybean mutant nts1007. Planta 222, 709-715

38 van Rhijn, P. et al. (1997) Expression of early nodulin genes in alfalfa mycorrhizae indicates that signal transduction pathways used in forming arbuscular mycorrhizae and Rhizobium-induced nodules might be conserved. Proc. Natl. Acad. Sci. U. S. A. 94, 5467-5472

39 Braunberger, P.G. et al. (1991) Effect of phosphorus nutrition on morphological characteristrics of vesicular-arbuscular mycorrhizal colonization of maize. New Phytol. 119, 107-113

40 Vierheilig, H. (2004) Further root colonization by arbuscular mycorrhizal fungi in already mycorrhizal plants is suppressed after a critical level of root colonization. J. Plant Physiol. 161, 339-341

41 Tenaillon, M.I. et al. (2004) Selection versus demography: a multilocus investigation of the domestication process in maize. Mol. Biol. Evol. 21, $1214-1225$

42 Tinker, P. and Nye, P. (2000) Solute Movement in the Rhizosphere, Oxford University Press

43 Zhu, Y-G. et al. (2003) Phosphorus efficiency and responses of barley (Hordeum vulgare L.) to arbuscular mycorrhizal fungi grown in highly calcereous soil. Mycorrhiza 13, 93-100

44 Benedetto, A. et al. (2005) Expression profiles of a phosphate transporter gene $(G \operatorname{mos} P T)$ from the endomycorrhizal fungus Glomus mosseae. Mycorrhiza 15, 620-627

45 Harrison, M.J. and v Buuren, M.L. (1995) A phosphate transporter from the mycorrhizal fungus Glomus versiforme. Nature 378 , 626-629

46 Maldonado-Mendoza, I.E. et al. (2001) A phosphate transporter gene from extra-radical mycelium of an arbuscular mycorrhizal fungus Glomus intraradices is regulated in response to phosphate in the environment. Mol. Plant Microbe Interact. 14, 1140-1148

47 Harrison, M.J. et al. (2002) A phosphate transporter from Medicago truncatula involved in the acquisition of phosphate released by arbuscular mycorrhizal fungi. Plant Cell 14, 2413-2429

48 Nagy, R. et al. (2005) The characterization of novel mycorrhiza-specific phosphate transporters from Lycopersicon esculentum and Solanum tuberosum uncovers functional redundancy in symbiotic phosphate transport in solanaceous species. Plant J. 42, 236-250

49 Glassop, D. et al. (2005) Cereal phosphate transporters associated with the mycorrhizal pathway of phosphate uptake into roots. Planta 222, 688-698

50 Maeda, D. et al. (2006) Knockdown of an arbuscular mycorrhizainducible phosphate transporter gene in Lotus japonicus suppresses mutualistic symbiosis. Plant Cell Physiol. 47, 807-817 